\title{
Large Strain Mechanical Behavior of 1018 Cold-Rolled Steel over a Wide Range of Strain Rates
}

\begin{abstract}
M. VURAL, D. RITTEL and G. RAVICHANDRAN
The large-strain constitutive behavior of cold-rolled 1018 steel has been characterized at strain rates ranging from $\dot{\varepsilon}=10^{-3}$ to $5 \times 10^{4} \mathrm{~s}^{-1}$ using a newly developed shear compression specimen (SCS). The SCS technique allows for a seamless characterization of the constitutive behavior of materials over a large range of strain rates. The comparison of results with those obtained by cylindrical specimens shows an excellent correlation up to strain rates of $10^{4} \mathrm{~s}^{-1}$. The study also shows a marked strain rate sensitivity of the steel at rates exceeding $100 \mathrm{~s}^{-1}$. With increasing strain rate, the apparent average strain hardening of the material decreases and becomes negative at rates exceeding $5000 \mathrm{~s}^{-1}$. This observation corroborates recent results obtained in torsion tests, while the strain softening was not clearly observed during dynamic compression of cylindrical specimens. A possible evolution scheme for shear localization is discussed, based on the detailed characterization of deformed microstructures. The Johnson-Cook constitutive model has been modified to represent the experimental data over a wide range of strain rates as well as to include heat-transfer effects, and model parameters have been determined for 1018 cold-rolled steel.
\end{abstract}

\section{INTRODUCTION}

THE determination of large strain constitutive behavior of materials is a key step toward accurate modeling of numerous processes such as plastic forming, plastic fracture, and high-speed penetration. Moreover, the behavior of the material should be determined over a large range of strain rates, as these are well known to influence the overall mechanical response. ${ }^{[1]}$ While a variety of techniques are available for this purpose, the constitutive behavior of a given material is often studied through the use of various specimens and experimental techniques. Here, one should mention the Kolsky apparatus (split Hopkinson pressure bar, Kolsky ${ }^{[2]}$ ) as the main experimental technique for the dynamic characterization of cylindrical specimens in the range of strain rates from $10^{2}$ to $10^{3}$ $\mathrm{s}^{-1}$. At strain rates of $10^{5} \mathrm{~s}^{-1}$ and above, the key technique is that of plate impact experiments, for uniaxial strain and pressure shear experiments. ${ }^{[3]}$ Shear testing at high strain rates has been carried out by means of a torsional Kolsky apparatus. ${ }^{[4]}$ Using this technique, Marchand and Duffy ${ }^{[5]}$ studied adiabatic shear band formation in HY100 steel (also Duffy and $\mathrm{Chi}^{\left[{ }^{[6]}\right.}$ on cold-rolled 1018 and martensitic steels). Gilat and $\mathrm{Wu}^{[7]}$ investigated 1020 hot-rolled steel over a wide range of temperatures and strain rates, with pure shear tests. Gilat and Cheng ${ }^{[8]}$ characterized the high rate shear behavior of 1100 aluminum and modeled the experimental setup using finite element analysis. To achieve large shear strains and strain rates, various specimen geometries were devised in which the linear displacement applied at the specimen boundary was trans-

M. VURAL, formerly Visiting Associate in Aeronautics, California Institute of Technology, Pasadena, CA 91125, is now Assistant Professor of Mechanical and Aerospace Engineering with the MMAE Department, Illinois Institute of Technology, Chicago, IL 60616. Contact e-mail: vural@ iit.edu G. RAVICHANDRAN, Professor of Aeronautical and Mechanical Engineering, is with the Graduate Aeronautical Laboratories, California Institute of Technology, Pasadena, CA 91125. Contact e-mail: mvural@ caltech.edu D. RITTEL, Associate Professor of Mechanical Engineering, is with the Faculty of Mechanical Engineering, Israel Institute of Technology, Technion, 32000 Haifa, Israel.

Manuscript submitted January 8, 2003. formed into local shear in the gage section. An example of such a specimen is the so-called "top hat specimen," which has been used by many researchers to investigate adiabatic shear band formation, e.g., Meyers et al.${ }^{[9]}$ Recently, Rittel et $a l .^{[10,11]}$ developed a framework for the large strain testing of materials over a wide range of strain rates, ranging from the quasi-static $\left(10^{-4} \mathrm{~s}^{-1}\right)$ to high strain rates $\left(10^{4} \mathrm{~s}^{-1}\right)$, using one kind of specimen. The key concept lies in an original specimen geometry by which shear dominant strain field is applied to the gage section through compressive loading, either in quasi-static or dynamic (Kolsky apparatus) mode. This specimen, the shear compression specimen (SCS), was validated through a preliminary investigation of OFHC (Oxygen-Free High Conductivity) copper at various strain rates. ${ }^{[10,11]}$

Regardless of the experimental technique, one should point out that all the large strain, high strain rate experiments are accompanied by heat generation (thermoplastic coupling, Taylor and Quinney ${ }^{[12]}$, which may cause a noticeable elevation of temperature, either homogeneously within the deforming gage or in a localized mode such as adiabatic shear banding (for a review, see, e.g., Bai and Dodd ${ }^{[13]}$ ). Temperature rise is likely to affect the overall mechanical response of the material by causing thermal softening that competes with the strain and strain rate hardening of the material (Meyers ${ }^{[1]}$ ).

In this article, we study the large strain mechanical behavior of cold-rolled 1018 steel, over a wide range of strain rates, ranging from quasi-static $\left(10^{-3} \mathrm{~s}^{-1}\right)$ up to high strain rates $\left(5 \times 10^{4} \mathrm{~s}^{-1}\right)$. The 1018 steel is a pearlitic mild steel $(0.18 \mathrm{wt}$ pct C), for which there is a relative scarcity of experimental data available in the literature in the high strain rate regime and is an excellent model material for carbon steels. Costin and Duffy ${ }^{[14]}$ characterized the fracture behavior of this steel over a variety of temperatures. They also included a description of the flow properties of the material tested in shear. Hartley et al..$^{[15]}$ investigated adiabatic shear band formation in cold-rolled 1018 steel, using torsion tests and infrared detectors to monitor transient temperature changes. Duffy and $\mathrm{Chi}^{\left[{ }^{[6]}\right.}$ used the same basic technique to investigate high strain rate behavior of several steels, including cold-rolled 1018 steel. 
Kapoor and Nemat-Nasser ${ }^{[16]}$ investigated the efficiency of the thermomechanical conversion in various materials, including cold-rolled 1018 steel. Finally, Cheng ${ }^{[17]}$ used high strain rate torsion tests to study the behavior of this material. In his experiments, the maximum shear strain rate reached $2.2 \times$ $10^{4} \mathrm{~s}^{-1}$. Throughout this work, emphasis is put on a comparison of SCS results with the available data in order to further assess the qualities and limitations of the newly developed SCS technique for constitutive testing.

The article is organized as follows: first, we recall some basic facts about the SCS and the data reduction technique, with some modifications. The experimental results are presented in Section III. In this section, a limited investigation of the effect of the SCS gage geometry on the measured flow properties is first discussed. Then, we present a detailed comparison between the results obtained with the SCS vs cylindrical geometries over a wide range of strain rates. Finally, a detailed microstructural account of the shear deformation patterns is presented. In Section IV, specific trends in the hardening behavior of the material observed at high strain rates are discussed in comparison with torsion and uniaxial compression tests. Then, a modification to the Johnson-Cook constitutive model ${ }^{[18]}$ is introduced to enlarge its applicable range of strain rate, and the parameters of the modified model are determined for 1018 steel based on the SCS data. A finite range of strain rate for transition from isothermal to adiabatic deformation process is also recognized and augmented into the modified model. A potential scheme for the evolution of shear localization is also discussed in this section. This section is followed by concluding remarks for the present study.

\section{EXPERIMENTAL PROCEDURE}

\section{A. Specimens and Testing}

Both SCS and cylindrical specimens were machined from a commercially obtained 12.7-mm-diameter cold-rolled 1018 steel bar, as shown in Figure 1. The 1018 steel was provided by
EMJ Co. (Los Angeles, CA) with the chemical composition by wt pet of $0.18 \mathrm{C}, 0.60$ to $0.90 \mathrm{Mn}, \max 0.04 \mathrm{P}, \max 0.05 \mathrm{~S}$. The experiments with shear compression specimens were performed over a range of strain rates from $1 \times 10^{-3}$ to $5 \times 10^{4} \mathrm{~s}^{-1}$. For comparison purposes, a set of cylindrical specimens was prepared and tested in the strain rate range, from $5 \times 10^{-4}$ to $1 \times 10^{4} \mathrm{~s}^{-1}$. Specimens were loaded by a screw-driven testing machine (Instron, model 4204, Canton, MA) at quasi-static strain rates, and by a Kolsky (split Hopkinson) pressure bar apparatus at varying strain rates over $10^{2} \mathrm{~s}^{-1}$.

The cylindrical specimens had a common diameter of $7.62 \mathrm{~mm}$. The quasi-static tests were performed with a length $(L)$-to-diameter $(D)$ ratio of 1 . However, three different L/D ratios $(\mathrm{L} / \mathrm{D}=1,1 / 2,1 / 4)$ were used for high-strain-rate testing of these specimens to achieve the highest possible strain rates. For shear compression tests, all the specimens used in both quasi-static and dynamic tests had a common diameter of $10 \mathrm{~mm}$ and variable gage thickness of $w=2.54,1.70$, 0.50 , and $0.25 \mathrm{~mm}$.

In quasi-static tests, a compression fixture was used to ensure that the loading rods were perfectly aligned with each other in order to minimize any unwanted shear forces on the specimen-loading rod interface. The specimen was sandwiched between the loading rods, and compression was applied by means of a screw-driven materials testing system. The deformation data of specimens were obtained from the crosshead displacement transducer, which was calibrated to account for the machine and fixture compliance.

For dynamic tests, the specimens were loaded using a Kolsky (split Hopkinson) pressure bar. The dimensions of the bars in the Kolsky pressure bar setup used in this study are 1215 and $1020 \mathrm{~mm}$ in length for the incident and transmission bars, respectively, with a common diameter of $19.05 \mathrm{~mm}$. The striker bars of 19.05-mm diameter were used with varying lengths to achieve desired pulse duration. All the bars are made of precision ground high-strength C350 maraging steel. The conventional Kolsky pressure bar technique $\left(K_{\text {Kolsky }}{ }^{[2]}\right.$ ) is by now a well-established classic
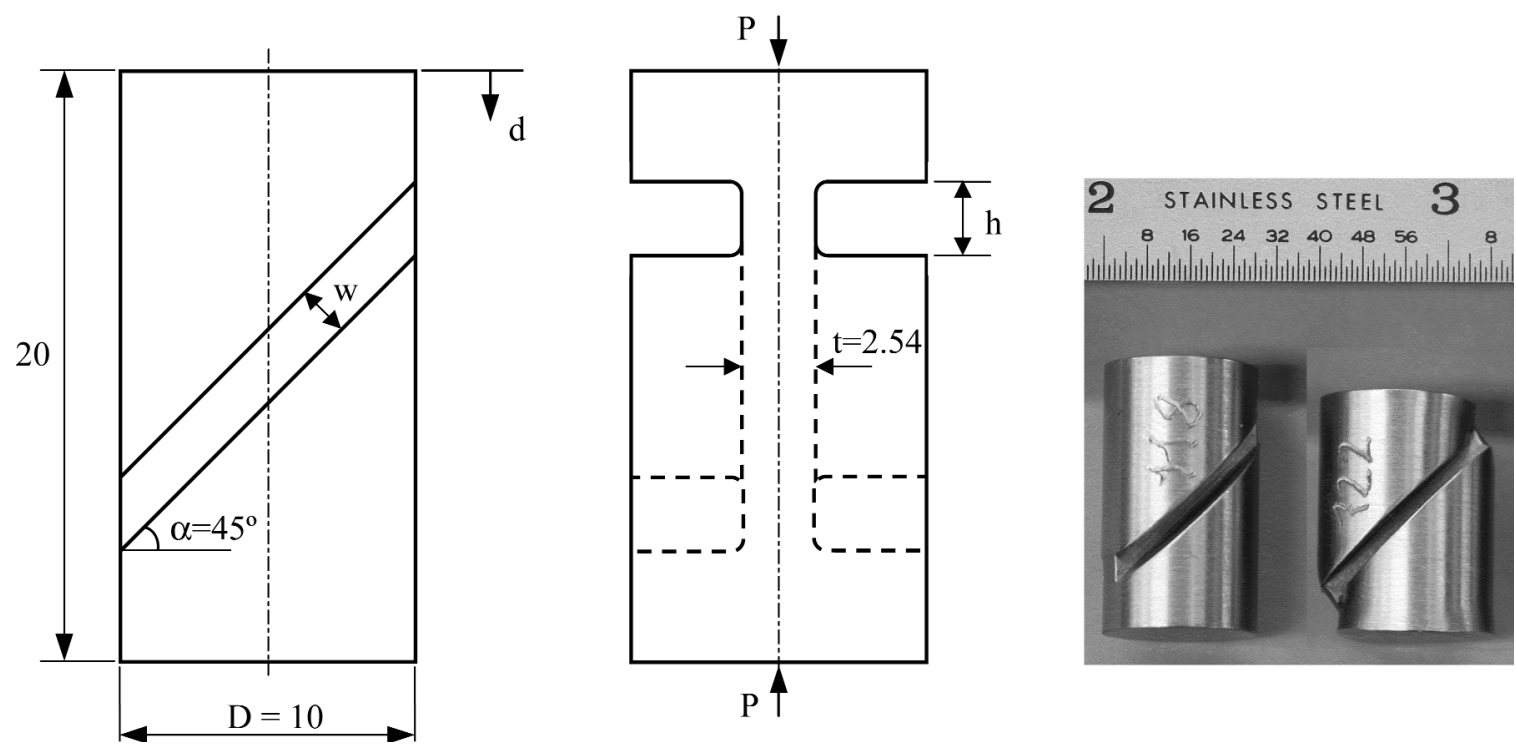

Fig. 1-Schematic representation of the SCS. All dimensions are in millimeters. The terms $D, h$, and $t$ are the geometrical parameters used for equivalent stress and equivalent strain determination. The photograph of two SCSs before and after deformation is also shown on the right for better visualization of specimen geometry. 
high-strain-testing technique for metals and the operational details can be found elsewhere (e.g., Gray $\left.{ }^{[19]}\right)$. In some of the dynamic tests, stop rings were used to limit the maximum strain experienced by specimens to predetermined strain levels and to investigate the microstructural evolution of deformation at the gage section. The stop ring is a hallow tube made of high-strength C350 maraging steel with a length slightly shorter than that of specimen and with an inner diameter sufficiently larger than that of the specimen but still smaller than that of bars. When the specimen, which is placed within the stop ring and between the incident and transmission bars, is compressed to the length of stop ring, the contact is established between the bars and the stop ring and a considerable portion of stress wave is transmitted to the transmission bar through the stop ring. From this point onward, the shortening rate of the specimen rapidly decelerates to almost zero, and, thus, the total strain in specimen is limited to a predetermined value that depends on the length of stop ring

\section{B. The SCS Technique}

The SCS is a cylindrical specimen with $45 \mathrm{deg}$ side grooves (Rittel et al. ${ }^{[10]}$ ), as shown in Figure 1. By imposing a compressive load, a shear dominant strain field develops in the gage section of the specimen. Yet, numerical analysis of this specimen showed that the state of stress in the gage section is three-dimensional, rather than shear only, as in the case of most specimen geometries with the exception of the case of torsion of thin walled tubes. Therefore, the strains and stresses can be reduced to equivalent strains and stresses in the Mises' sense. The simplified relations between displacement, load, geometrical parameters, and equivalent strain $\left(\varepsilon_{e q}\right)$ and stress $\left(\sigma_{e q}\right)$ were devised as follows:

$$
\begin{gathered}
\varepsilon_{e q}=\frac{d}{h} ; \quad \dot{\varepsilon}_{e q}=\frac{\dot{d}}{h} \\
\sigma_{e q}=k_{1}\left(1-k_{2} \varepsilon_{e q}\right) \frac{P}{D t}
\end{gathered}
$$

where

$d=$ prescribed displacement (Figure 1),

$h=$ gage height (Figure 1),

$P=$ applied load (Figure 1),

$D=$ specimen diameter (Figure 1),

$t=$ gage thickness (Figure 1),

$k_{1}=$ constant $\left(0.85\right.$ in Rittel et al..$\left.^{[10,11]}\right)$, and

$k_{2}=$ constant $\left(0.20\right.$ in Rittel et al..$\left.^{[10,11]}\right)$.

In the present experiments, two slight changes were brought to the aforementioned formulas. First, $k_{1}$ was taken to be 1 , rather than the originally proposed value of 0.85 (Rittel et $\left.a l^{[10,11]}\right)$. The reason for that was an observed improved fit between cylindrical and SCS specimen results. This value suggests the existence of a simple stress state in the gage section, such as that shown in Appendix A, for which the equivalent von Mises stress requires $k_{1}=1$. In addition, when the gage width $(w)$ reaches $0.5 \mathrm{~mm}$ and below, the gage experiences a state of strain that is close to that of the pressureshear experiments (Clifton and Klopp ${ }^{[3]}$ ). Consequently, there is no need to correct for three-dimensional strain effects, as shown in Eq. [2]. In this case, when $w \leq 0.5 \mathrm{~mm}$, we also set $k_{2}=0$. It should be mentioned that very thin gage sections of this kind were not tested in previous work.

\section{RESULTS}

\section{A. The Influence of the Gage Width (w)}

Results of tests carried out at a fixed strain rate of $10^{-2} \mathrm{~s}^{-1}$ with various gage widths are shown in Figure 2. These results clearly show that when the gage width is varied by one order of magnitude, the equivalent stress-strain curves determined using Eqs. [1] and [2] are still very similar and close to each other. At this point, one can conclude that, while the gage width should certainly be taken into account by modifying the basic Eqs. [1] and [2], this factor does not strongly influence the results. This observation validates the choice of the factor $k_{2}$, as it also opens the way for investigating material behavior at larger strains and higher strain rates than those previously thought possible with the SCS geometry.

\section{B. Mechanical Behavior at Various Strain Rates}

The flow curves (true stress-true strain) that were determined over a wide range of strain rates are shown in Figure 3. This figure covers a wide range of strain rates from quasi-static to dynamic regime. For each class of strain rates, we have included the results obtained using cylindrical specimens. For these specimens, the attainable maximum strain is limited by barreling at strains of approximately 0.4 . Moreover, at very high strain rates, above $10^{4} \mathrm{~s}^{-1}$ as in Figure 3(f), one cannot use cylindrical specimens so that the comparison is no longer possible. From this figure, it first appears that there is a high degree of agreement between the results of SCSs and cylindrical specimens, irrespective of the strain rate, and the yield stress is observed to increase with the strain rate for both kinds of specimens.

However, when the strain rate exceeds $5 \times 10^{3} \mathrm{~s}^{-1}$, there is an obvious discrepancy between the stress-strain curve of cylindrical specimens and that of SCS. The cylindrical specimen exhibits little, if no, strain hardening at all. By contrast, the SCS shows a clear strain softening effect that increases

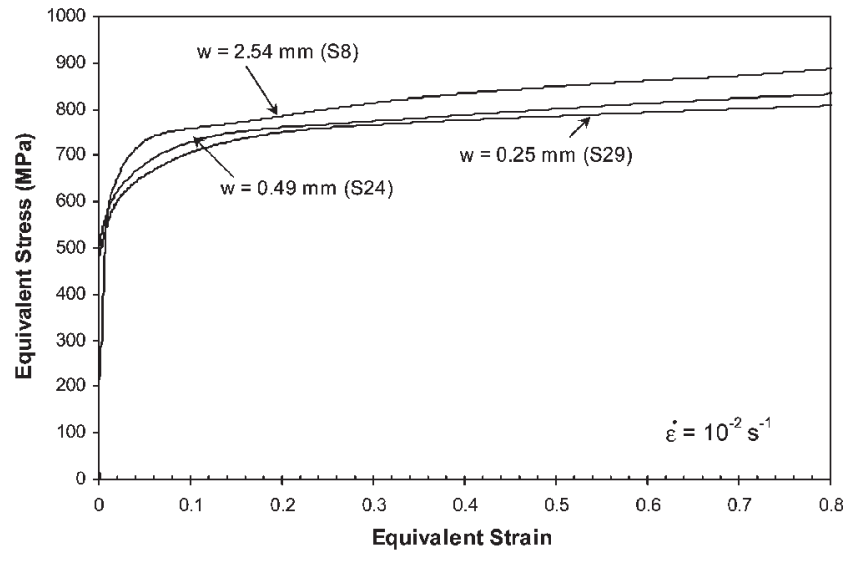

Fig. 2-Effect of the gage width $(w)$ on the flow behavior of 1018 steel shear compression specimens (SCS). Note that the variation of $w$ over one order of magnitude has a relatively small effect on the stress-strain characteristics. 

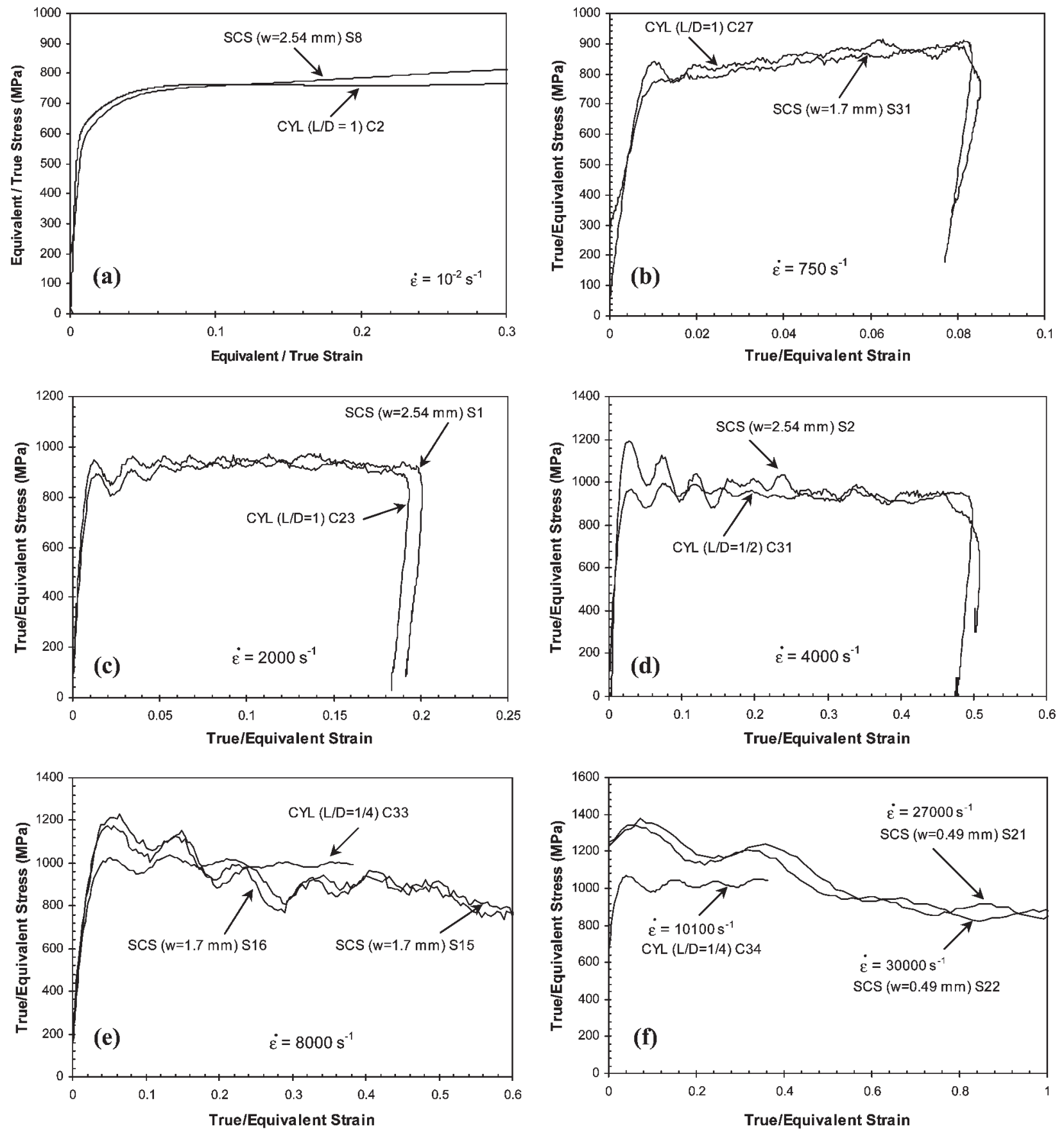

Fig. 3-Comparison of large strain behavior of 1018 steel at various strain rates, as determined by using cylindrical (CYL) and SC specimens. Note the excellent agreement up to $\dot{\varepsilon}=4000 \mathrm{~s}^{-1}$. At higher strain rates, SCSs exhibit noticeable strain softening as opposed to cylindrical specimens.

with the strain rate, beyond about $5 \times 10^{3} \mathrm{~s}^{-1}$. This subject will be addressed in detail in the next section.

The influence of strain rate on the flow behavior of 1018 steel can be further assessed by considering the flow stress at a selected level of plastic strain $\varepsilon_{p}=0.10$. Figure 4 combines results obtained for both cylindrical and SCS specimens. Once again, one can note the very good agreement between two different techniques and specimen geometries. It also appears that the flow stress is relatively insensitive to changes in strain rate, for rates of up to $10^{2} \mathrm{~s}^{-1}$. However, beyond this rate, there is a dramatic increase in the flow stress that eventually reaches a value of twice its quasi-static counterpart. Therefore, at high strain rates, cold-rolled 1018 steel reaches the quasi-static flow stress level of alloyed steels.

\section{Microstructural Characterization}

Metallographic longitudinal midsections of selected SCSs were prepared by electrodischarge machining. The specimens were subsequently prepared for microstructural characterization using standard metallographic techniques. Figure 5 shows the typical microstructure of two specimens that have 
been loaded in the Kolsky pressure bar at a strain rate of around $10^{4} \mathrm{~s}^{-1}$ to the predetermined strains $(0.45$ and 0.90 , respectively) using stop rings. The microstructure is typical of pearlitic steels. Outside the gage section, the material is essentially in its original state, and one can note the significant amount of cold work in the starting material, as indicated by the aligned pearlite colonies. The shear deformation appears to be rather homogeneous in the gage section of these two specimens. The specimen shown in Figure 6 exhibits well-defined localization bands at the two transition regions between the gage section and the undeformed

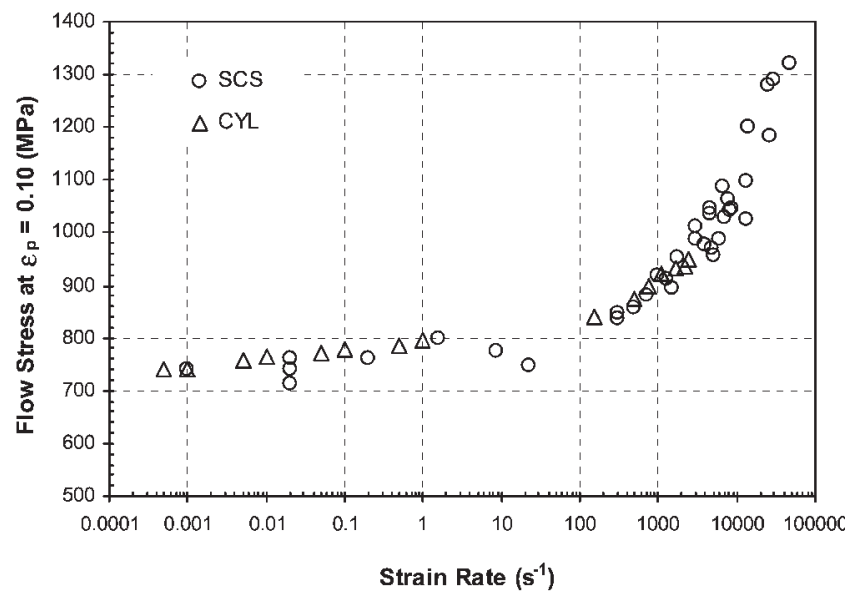

Fig. 4-Flow stress at $\varepsilon_{p}=0.10$ as a function of the strain rate. Note the very good agreement between cylindrical and SCS geometries. material. These two highly deformed bands, each of which is about $200-\mu \mathrm{m}$ wide, reduce the effective gage width. Finally, Figure 7 shows severe localization for two specimens, where one of the specimens is fractured along the localized shear band. The localization consists of both transition region localization, as in Figure 6, and secondary multiple shear band formations inside the gage section, giving it a wavy appearance. It can be noted that the width of the transition shear bands has shrunk dramatically, to less than $50 \mu \mathrm{m}$. In these bands, the shear deformation appears to have noticeably increased to a point where the fiberlike elongated grains in the rolling direction are now parallel to the orientation of gage section. The secondary shear bands are narrow and evenly distributed. They are similar to shear bands observed in hot-rolled steel by $\mathrm{Xu}$ et al. ${ }^{[20]}$ In Figure 8 , the fractured specimen's microstructure is shown and a clear correlation can be observed between the fracture surface steps and the aforementioned secondary shear bands.

The microhardness profile across the band is shown in Figure 9 for the specimen shown in Figure 6. As expected, the hardness varies with the amount of deformation, and the various spatial domains, i.e., gage section and its two outer localized bands, are well captured.

\section{DISCUSSION}

The results obtained so far are of a dual nature. First, the very good agreement between various specimen geometries and experimental methods shows that the SCS method is an additional reliable method, with the advantage that
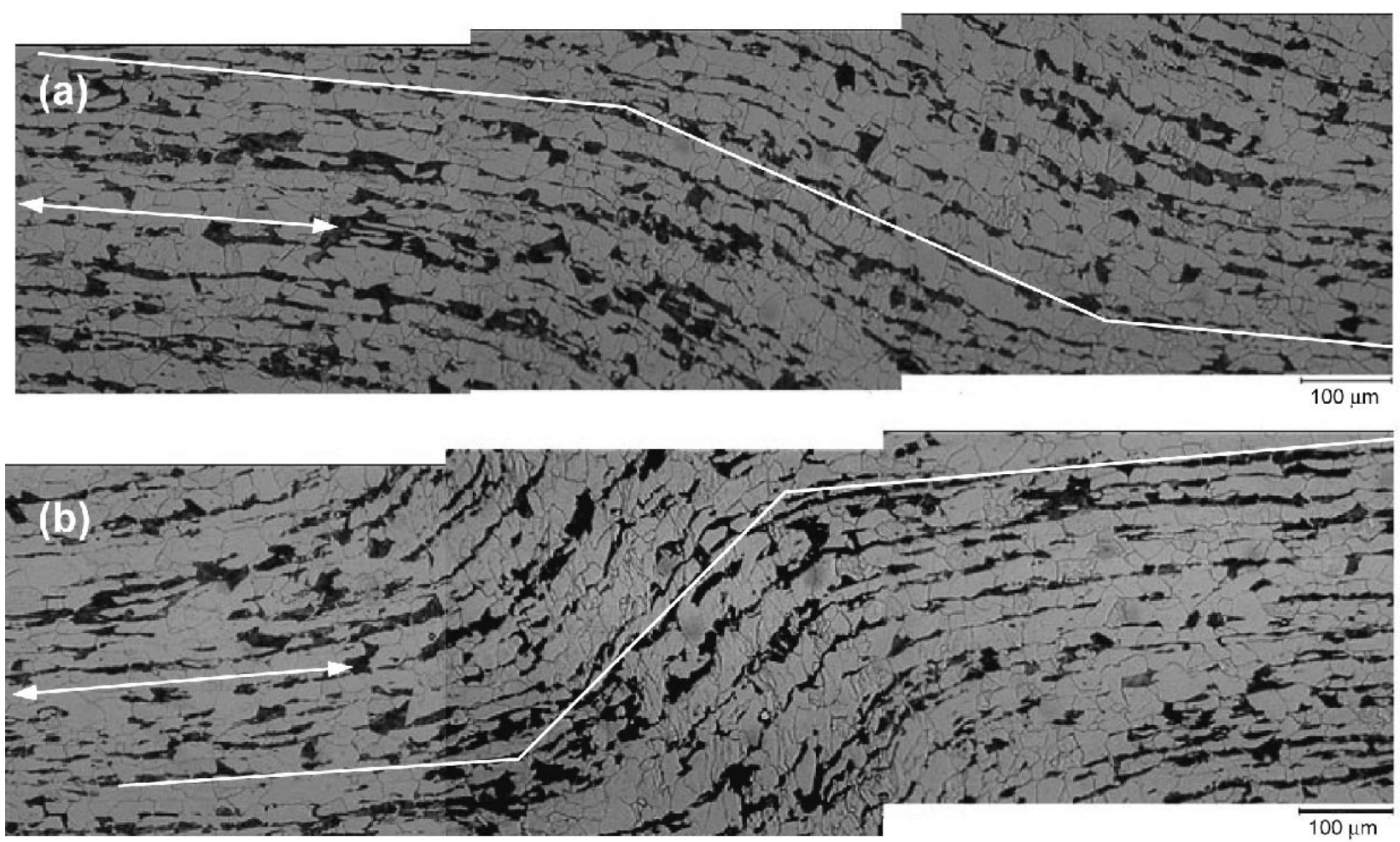

Fig. 5-Typical micrographs of the homogeneous deformation in gage section for SCS dynamically compressed to predetermined strains $\left(\varepsilon_{T}\right)$ : $(a) \varepsilon_{T}=0.45$, $\dot{\varepsilon}=1.4 \times 10^{4} \mathrm{~s}^{-1}, w=0.50 \mathrm{~mm}$; and $(b) \varepsilon_{T}=0.90, \dot{\varepsilon}=1.3 \times 10^{4} \mathrm{~s}^{-1}, w=0.25 \mathrm{~mm}$ (etchant is nital 3 pct). The contrast between dark pearlite colonies and light ferrite regions shows the shear-dominant deformation in gage section. The white lines following the path of pearlite colonies clearly show the shear deformation in the gage section. The direction of loading (and thus the specimen axis) is also shown in the micrographs with the white arrows 

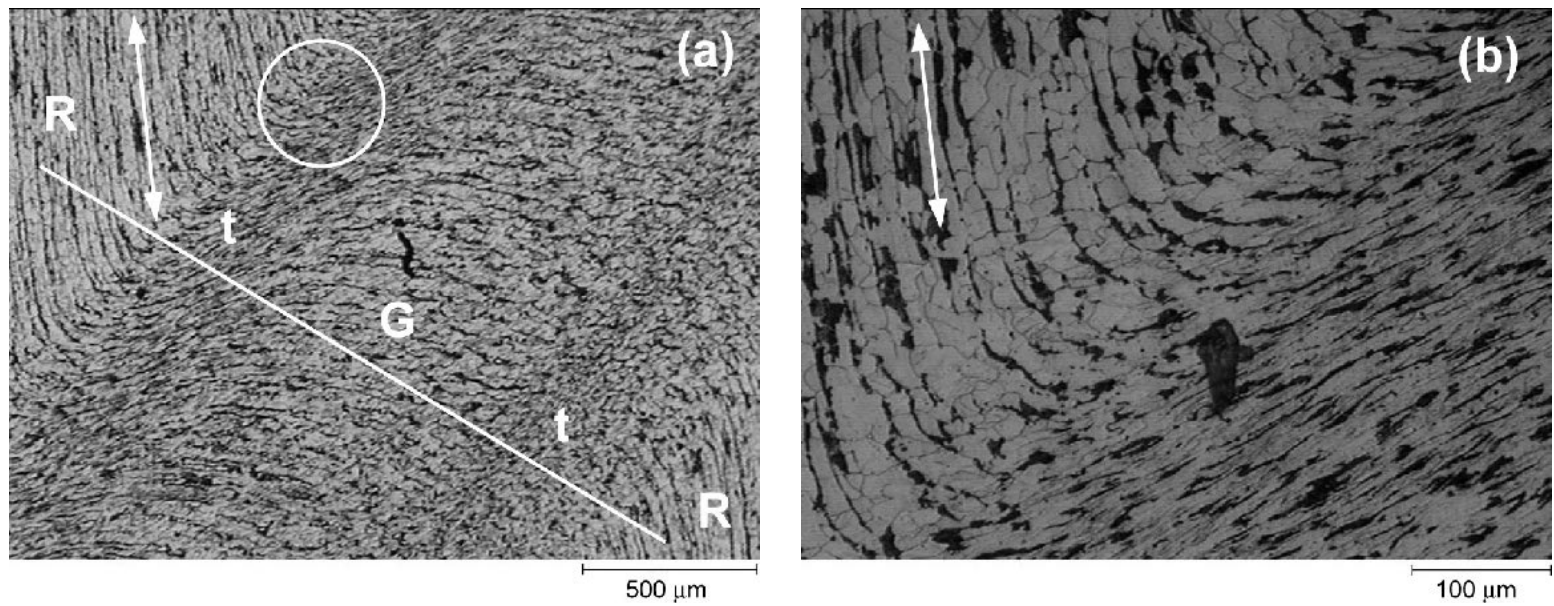

Fig. 6- (a) Typical micrographs of the localized bands in transition region $(t)$ between the undeformed part of specimen (R) and the gage section $(\mathrm{G})$ for $\varepsilon_{T}=0.55, \dot{\varepsilon}=4.5 \times 10^{3} \mathrm{~s}^{-1}, w=2.54 \mathrm{~mm}$. (b) The magnified view of localization in transition region, which is denoted by a circle in (a) (etchant is nital $3 \mathrm{pct})$. The arrows show the axis of SCS.

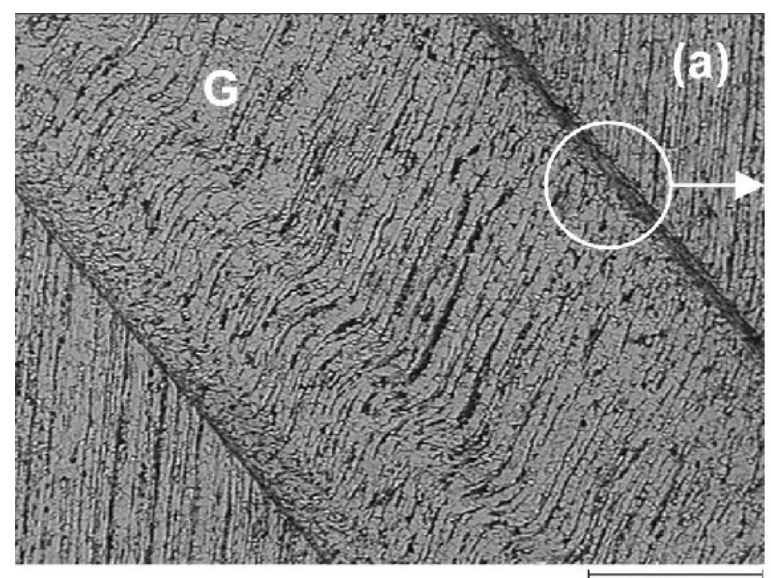

$500 \mu \mathrm{m}$

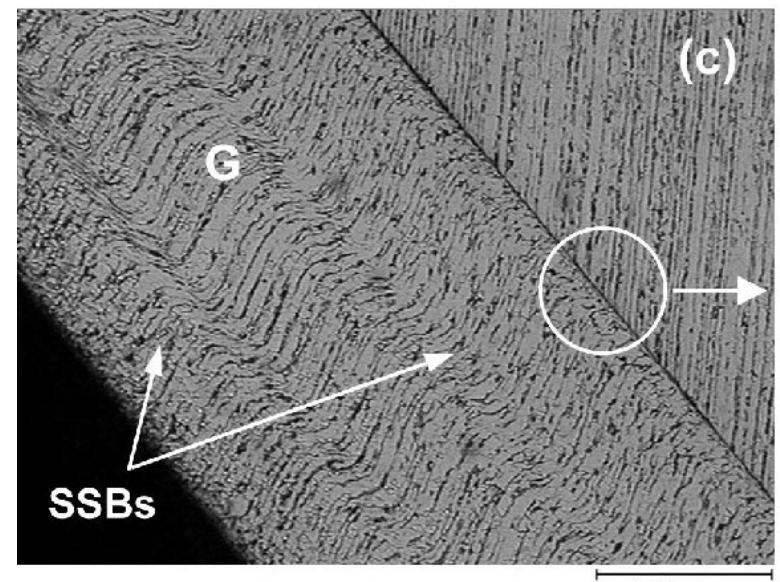

$500 \mu \mathrm{m}$

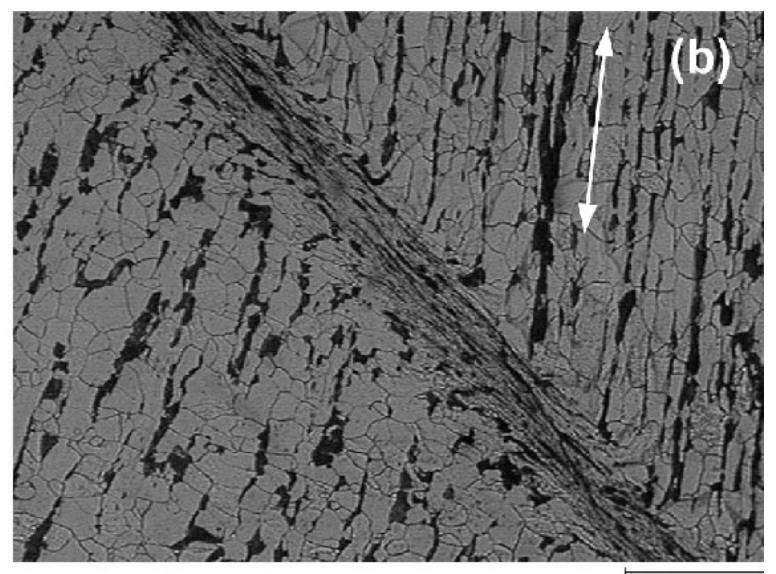

$100 \mu \mathrm{m}$

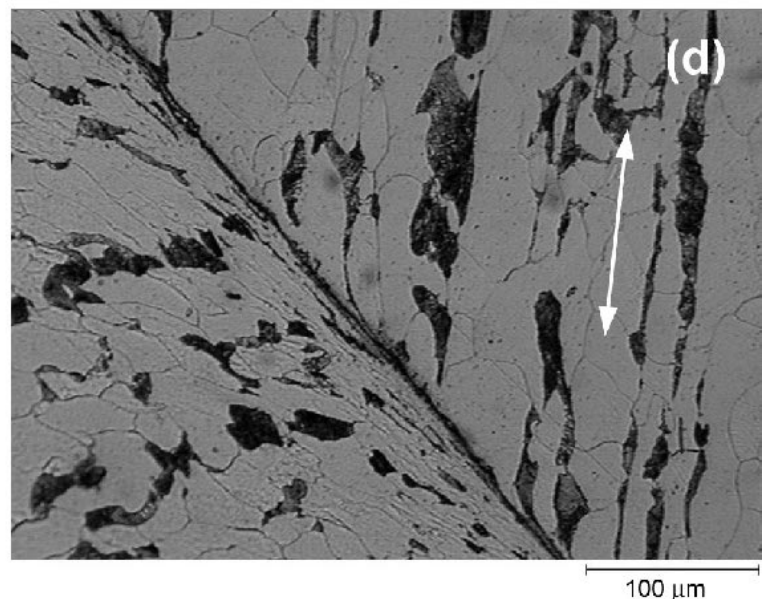

Fig. 7-Severe localization in the transition region and the secondary multiple shear band formations (SSBs) within the gage section for $(a)$ and $(b) \varepsilon_{T}=0.55$, $\dot{\varepsilon}=4.5 \times 10^{3} \mathrm{~s}^{-1}, w=1.70 \mathrm{~mm}$; and $(c)$ and $(d) \varepsilon_{T}=0.75, \dot{\varepsilon}=8 \times 10^{3} \mathrm{~s}^{-1}, w=1.70 \mathrm{~mm}$. Micrographs (b) and (d) are the magnified views of the encircled areas shown on their left and the arrows show the axis of SCSs (etchant is nital 3 pct).

the specimen is relatively simple to manufacture and test, in comparison with torsion tests. Moreover, this specimen opens a way to reach higher strain rates up to $10^{5} \mathrm{~s}^{-1}$ and, thus, bridge the traditional gap between Kolsky (split Hopkinson) bar testing and plate impact experiments. The present study has extended the simple formulas used to determine the equivalent stress and strains, to include very narrow gage width reaching $0.25 \mathrm{~mm}$. These observations corroborate previous observations and conclusions reached in prior work..$^{[10,11]}$ 


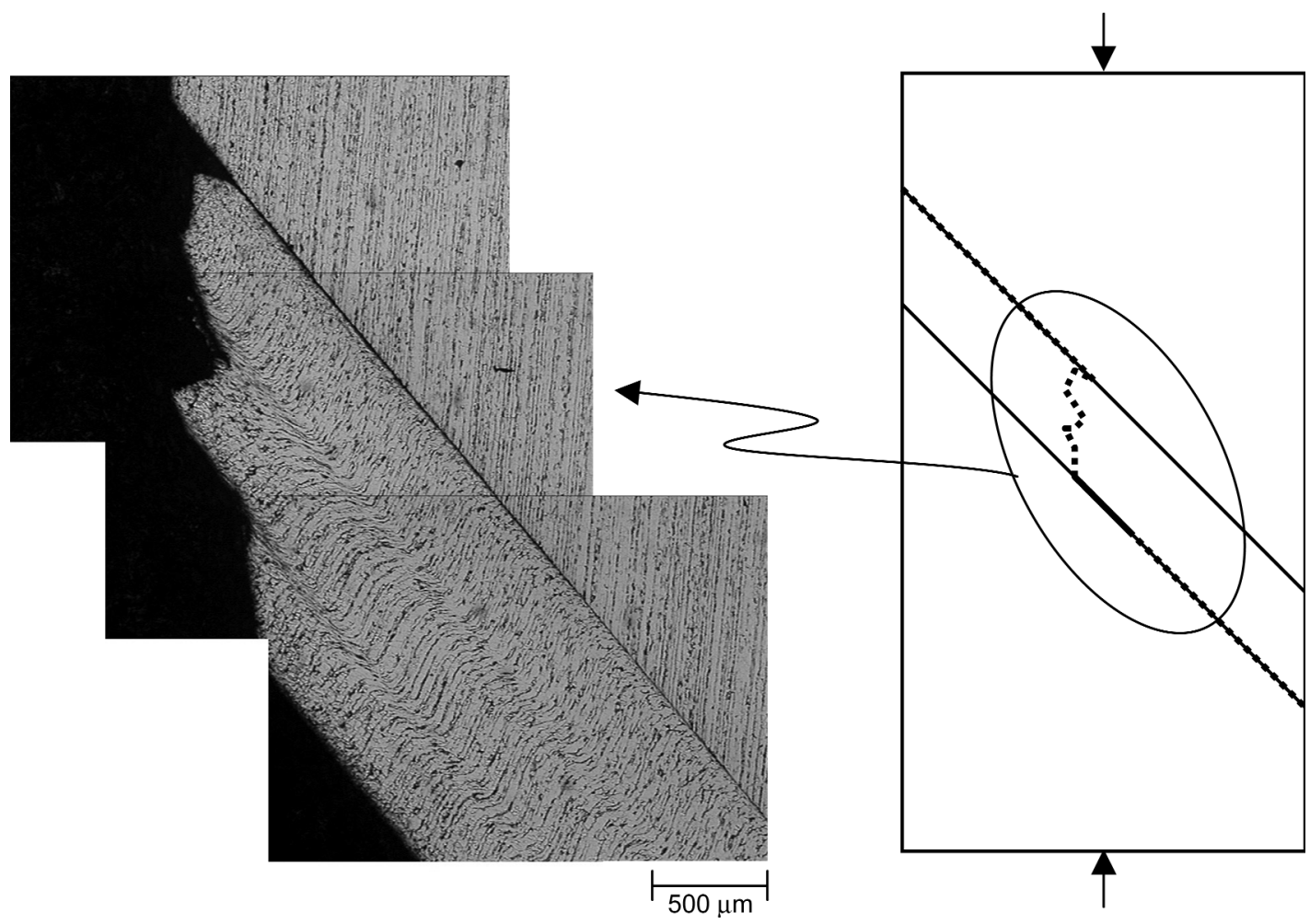

Fig. 8- Schematic of the specimen geometry and the typical failure path (dotted bold line) for fractured specimens (right) and the corresponding micrograph (left), where $\varepsilon_{T}=0.75, \dot{\varepsilon}=8 \times 10^{3} \mathrm{~s}^{-1}, w=1.70 \mathrm{~mm}$. Note the correlation between the wavy fracture pattern and the secondary shear bands (etchant is nital 3 pct).

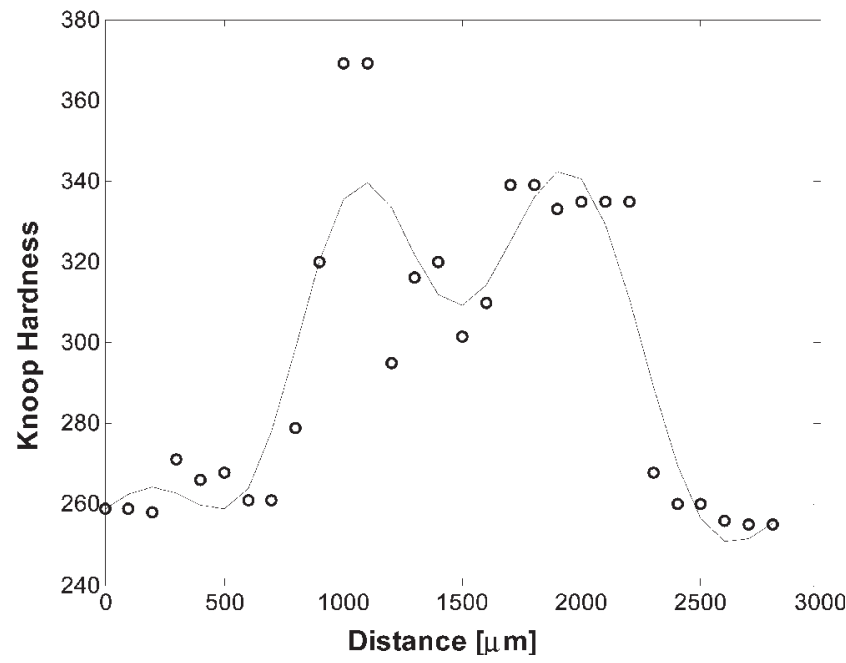

Fig. 9-Variation of microhardness along the gage section of the specimen shown in Fig. 6(a). The solid line represents a smoothed fit to data points. Note that the value $260 \mathrm{HK}$ corresponds to the hardness of undeformed base material out of gage section, and the double-peak structure around $340 \mathrm{HK}$ corresponds to the localization bands at two well-defined transition regions within the gage section shown in Fig. 6(a).

The second aspect of the study relates to the material itself, which will now be addressed. This is the first largescale comparative study, using also the SCS technique, applied to 1018 steel. The results obtained here confirm a general trend observed in many metallic materials, for the flow strength to increase with the strain rate (e.g., Campbell and Ferguson, ${ }^{[21]}$ Meyers $\left.^{[1]}\right)$. This trend has been observed over the entire range of strain rates investigated in this study. It should be noted that since the results shown in Figure 4 address the flow stress at relatively small plastic strains $\left(\varepsilon_{p}=0.10\right)$, thermomechanical coupling can be considered to be minimal. However, this effect is expected to become significant at large plastic strains.

A striking result obtained here is that SCS and cylinders yield very similar flow characteristics as long as the strain rate does not exceed $5 \times 10^{3} \mathrm{~s}^{-1}$. At higher strain rates, the SCS clearly shows a strain softening effect that does not appear with cylindrical specimens (Figures 3(e) and (f)). This discrepancy observed in the flow behavior essentially arises from the complexities in the compression of cylindrical specimens because of friction on the contacting surfaces and resulting complications such as the barreling and loss of uniaxial stress state in the specimen. Furthermore, it should be noted that the L/D ratio of cylindrical specimens is decreased to $1 / 4$ to achieve strain rates over $5 \times 10^{3} \mathrm{~s}^{-1}$, and, therefore, frictional effects are expected to become larger for these shorter specimens. To gain additional insight, we compare our results with the detailed results of Cheng, ${ }^{[17]}$ who performed torsion tests on 1018 steel. The equivalent stresses and strains for the SCS have been converted into shear stresses $(\tau=\sigma / \sqrt{3})$ and shear strains $(\gamma=\sqrt{3} \varepsilon)$, using Mises equivalents. Figure 10 shows the two sets of results, from torsion and SCS geometries. This comparison clearly shows that, for comparable shear strain rates, both techniques exhibit very similar flow characteristics, including a noticeable trend for strain softening at high strain rates. The fact that the torsion tests are free from frictional end effects and the good agreement between torsion and SCS techniques obviously indicate that SCS geometry overcomes the 

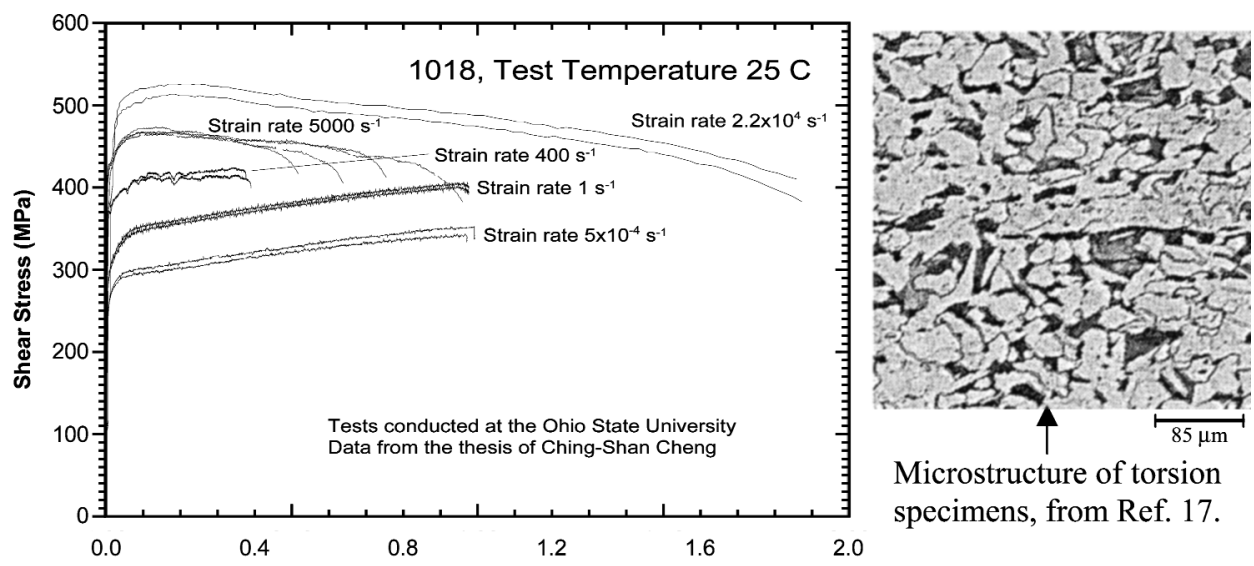

Microstructure of torsion specimens, from Ref. 17.

(a)
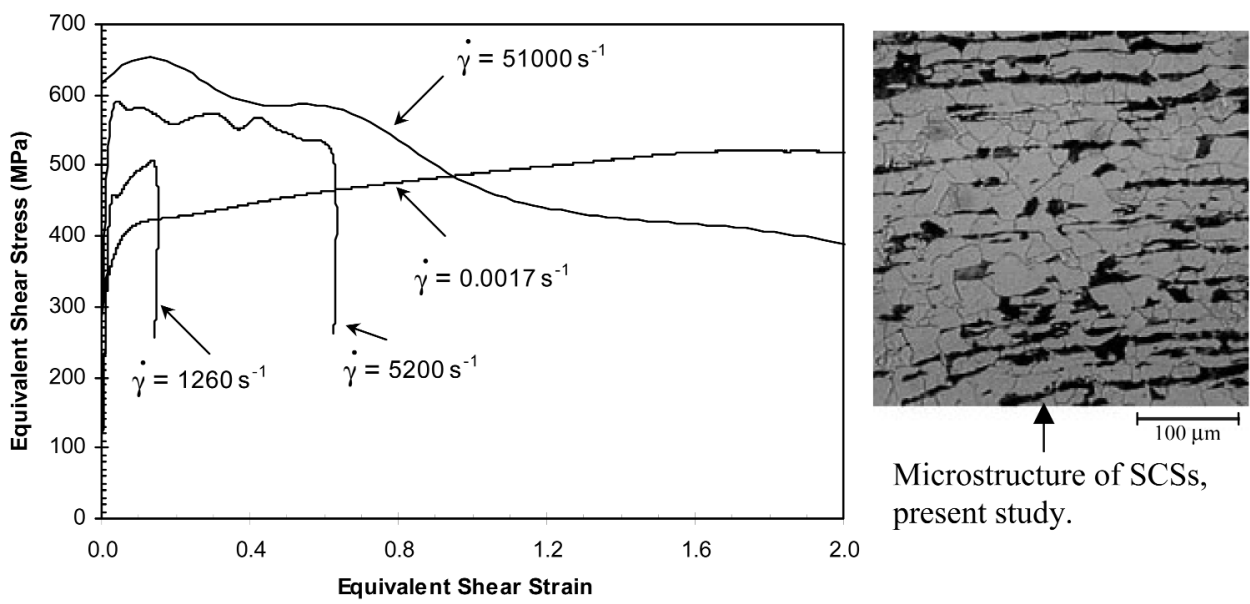

Microstructure of SCSs, present study.

(b)

Fig. 10 - Comparison of two testing techniques: (a) torsion tests (courtesy of A. Gilat, Ohio State University) vs (b) SCS technique after conversion to shear stress strain. Note the high level of similarity in the results, particularly in terms of hardening and softening trends as a function of strain rate. Specifically, torsion test shows strain softening at a strain rate of $5000 \mathrm{~s} \mathrm{~s}^{-1}$, which is in accord with the SCS test at $5200 \mathrm{~s}^{-1}$. The difference in the initial level of cold work in torsion and SCSs can be seen from the micrographs given next to the stress-strain curves and is the main reason for the difference observed in flow stress.

limitations traditionally encountered in cylindrical specimens and offers the potential of reaching higher strain rates that exceed $10^{4} \mathrm{~s}^{-1}$ threshold for the split Hopkinson bar system. Careful comparison of the flow curves reveals that the material tested by Cheng ${ }^{[17]}$ is softer than the material tested in the present study. This difference can be explained in terms of starting material's condition. The specimens used in the present study were machined from a 12.7-mm-diameter bar as opposed to larger diameter specimens in Cheng's work. ${ }^{[17]}$ Accordingly, a comparison of the two materials' microstructures shown in Figure 10 clearly reveals a higher degree of initial cold work in the material of the present study, corresponding to a higher initial hardness level for the larger amount of cold work.

Two factors can be invoked as contributing to strain softening that has been observed at high strain rates. The first relates to thermal softening, as a result of internal heat generation, i.e., adiabatic heating due to dissipation of plastic work into heat. This effect competes with the strain and strain-rate hardening effect, and becomes gradually dominant at increasing strain rates. One can estimate the temperature rise, $\Delta T$, that develops in the gage of the specimen by assuming that a significant part, $\beta$, of the mechanical work gets converted into heat in a supposedly adiabatic deformation process,

$$
\Delta T=\eta \frac{\beta}{\rho C_{p}} \int_{0}^{\varepsilon_{p}} \sigma d \varepsilon
$$

in which $\beta$ is the fraction of plastic work converted into heat: ${ }^{[16,22,23]} \eta$ is a factor related to transient heat transfer and describes the fraction of heat retained in the body $(\eta=0$ and 1 for isothermal and adiabatic processes, respectively); $\sigma$ and $\varepsilon$ are the equivalent flow stress and plastic strain; and $\rho$ and $C_{p}$ are the density and heat capacity, respectively. For 1018 steel, $\rho=7870 \mathrm{~kg} / \mathrm{m}^{3}$ and $C_{p}=486 \mathrm{~J} / \mathrm{kg} \mathrm{K}$. In Eq. [3], the factor $\beta$ is assumed to be a constant, 0.9 , the typical value assumed in the literature. First, let us consider in Figure 10(b) the SCS specimen tested at $\dot{\gamma}=5.2 \times 10^{3} \mathrm{~s}^{-1}$. Here, the flow stress level is taken as approximately constant and equal to $560 \mathrm{MPa}$ and the maximum strain $\gamma_{\max }=0.63$, which corresponds to an equivalent flow stress of $970 \mathrm{MPa}$ and maximum equivalent strain of 0.36 . Equation [3] yields an estimate of the temperature rise of $\Delta T=87 \mathrm{~K}$. Next, consider the SCS specimen tested at $\dot{\gamma}=5.1 \times 10^{4} \mathrm{~s}^{-1}$. (Figure 10(b)). Here, we assume an approximately constant 
flow stress level of $480 \mathrm{MPa}$ and a maximum strain of $\gamma_{\max }=2$. The corresponding calculated temperature rise will be $\Delta T=226 \mathrm{~K}$. These are macroscopically averaged estimates that do not take into account the possible localization of shear in the form of shear bands (e.g., Figure 6). It should thus be noted that the first estimate of $\Delta T=87 \mathrm{~K}$ is in very good agreement with the data quoted by Kapoor and Nemat$\operatorname{Nasser}^{[16]}(\Delta T=73$ to $90 \mathrm{~K})$ for 1018 steel at a similar equivalent strain and strain rate. The second estimate of $\Delta T=$ $226 \mathrm{~K}$ cannot be compared with available data at a similar strain rate. One should nevertheless note that a similar temperature rise was measured by Hartley et al., ${ }^{[15]}$ at much smaller strain rates not exceeding $\dot{\gamma}=1200 \mathrm{~s}^{-1}$, for most of their tests that did not end by fracture. These results are not contradictory if one notes that Kapoor and Nemat-Nasser ${ }^{[16]}$ measured the average temperature of a 1-mm-diameter area, whereas Hartley et al. ${ }^{[15]}$ measured the temperature of much narrower spots (250 to $20 \mu \mathrm{m})$, thus including the contribution of hot shear bands. However, taking the melting temperature of 1018 steel as $T_{m}=1773 \mathrm{~K}$, a temperature rise of $\Delta T=226 \mathrm{~K}$, above room temperature $(298 \mathrm{~K})$, does not exceed $0.3 T_{m}$. This result is quite different from that of $0.56 T_{m}$ obtained for OFHC copper (Rittel et al. ${ }^{[11]}$ ).

The contribution of thermal softening can be further assessed by considering that the overall material response can be represented by the Johnson-Cook constitutive model, ${ }^{[18]}$ which is a widely used phenomenological model:

$$
\sigma=\left(\sigma_{0}+B \varepsilon^{n}\right)\left(1+C \ln \frac{\dot{\varepsilon}}{\dot{\varepsilon}_{0}}\right)\left(1-T_{*}^{p}\right)
$$

where

$$
T_{*}=\frac{T-T_{r}}{T_{m}-T_{r}}
$$

where the $\sigma_{0}, B, n, C$, and $p$ are model parameters, $\dot{\varepsilon}_{0}$ is a reference strain rate at which the yield stress is $\sigma_{0}$, and the subscripts $r$ and $m$ in Eq. [4b] indicate reference (ambient) and melting temperatures, respectively. The Johnson-Cook model given in Eq. [4] provides a satisfactory prediction of flow stress for large strains and high strain rates when its dependence on strain rate is linear in semilogarithmic scale. However, the experimental data presented in Figure 4 do not follow a linear trend but can be considered to have powerlaw dependence on strain rate in two subsequent regions, with a transition strain rate at about $\dot{\varepsilon}=10^{2} \mathrm{~s}^{-1}$. In order to account for this nonlinear behavior, the Johnson-Cook model has been modified so as to recognize the strain rate dependence of the parameters $C$ and $\dot{\varepsilon}_{0}$ as elaborated in Appendix B. The model parameters of the modified Johnson-Cook equation can be found in Table I for 1018 cold-rolled steel. The modified model predicts a softening effect of 21 to 36 pct for the two aforementioned temperature rises ( 87 and $226 \mathrm{~K}$ ), respectively.

Table I. Parameters of the Modified Johnson-Cook model for 1018 Cold-Rolled Steel

\begin{tabular}{lcccccc}
\hline$\sigma_{0}(\mathrm{MPa}) *$ & $B(\mathrm{MPa})$ & $n$ & $n_{1}$ & $n_{2}$ & $\dot{\varepsilon}_{t}\left(\mathrm{~s}^{-1}\right)$ & $p$ \\
\hline 560 & 300 & 0.32 & 0.007 & 0.075 & 96 & 0.55 \\
\hline *Yield stress at a reference strain rate $\dot{\varepsilon}_{01}$ & $=5 \times 10^{-6} \mathrm{~s}^{-1}$. \\
\hline
\end{tabular}

These estimates show that the thermal softening effect is significant at very high strain rates, and of course in the case of a highly concentrated shear deformation.

Figure 11 shows the good correlation between experimental data and the predictions of the modified Johnson-Cook model for the flow stress at an equivalent plastic strain of $\varepsilon_{p}=$ 0.10. The distinction between two model predictions in Figure 11 comes from the choice of factor $\eta$ in Eq. [3]. In the modified Johnson-Cook model (denoted by solid squares in Figure 11), $\eta$ is assumed to be 0 for quasi-static loading rates (isothermal) and 1 for dynamic loading rates (adiabatic) with a sharp transition at the strain rate of $\dot{\varepsilon}_{0}=10^{2} \mathrm{~s}^{-1}$. This sharp transition in $\eta$ manifests itself in Figure 11 as a sudden drop in the predicted flow stress at around $\dot{\varepsilon}_{0}=10^{2} \mathrm{~s}^{-1}$ due to the thermal softening effect. However, in reality, the transition from isothermal to adiabatic deformation conditions does not occur as a sudden jump but follows a gradual trend. During plastic deformation at quasi-static low strain rates, the characteristic time for heat diffusion is very small compared to the test duration and, therefore, heat generated due to plastic work is diffused into surroundings (mainly by conduction through loading platens) without any significant temperature rise in the deforming body. As the strain rate increases so does the rate of heat generation, and the rate of heat loss gradually becomes insufficient to diffuse the heat generated, resulting in a gradual temperature rise in the deforming body. At sufficiently high strain rates, the ratio of the rates of heat generation to heat loss becomes large enough to practically assume that the deformation process is adiabatic and all of the plastic work converted to heat is used to build up an adiabatic body temperature. In fact, Dixon and Parry ${ }^{[24]}$ observed this type of a gradual transition in their temperature measurements on uniaxially compressed carbon steel specimens. They detected a gradual transition from isothermal to adiabatic conditions in the range of strain rates from $\dot{\varepsilon}_{0}=10^{-2}$ to $10 \mathrm{~s}^{-1}$, spanning a range of three orders of magnitude. In order to account for the aforementioned transition effect, the factor $\eta$ in Eq. [3] is redefined as a function of strain rate, as follows:

$$
\eta=S\left(\dot{\varepsilon}, 0,1, \dot{\varepsilon}_{A}\right)=\frac{1}{2}\left[1+\tanh \left(s_{A} \ln \frac{\dot{\varepsilon}}{\dot{\varepsilon}_{A}}\right)\right]
$$

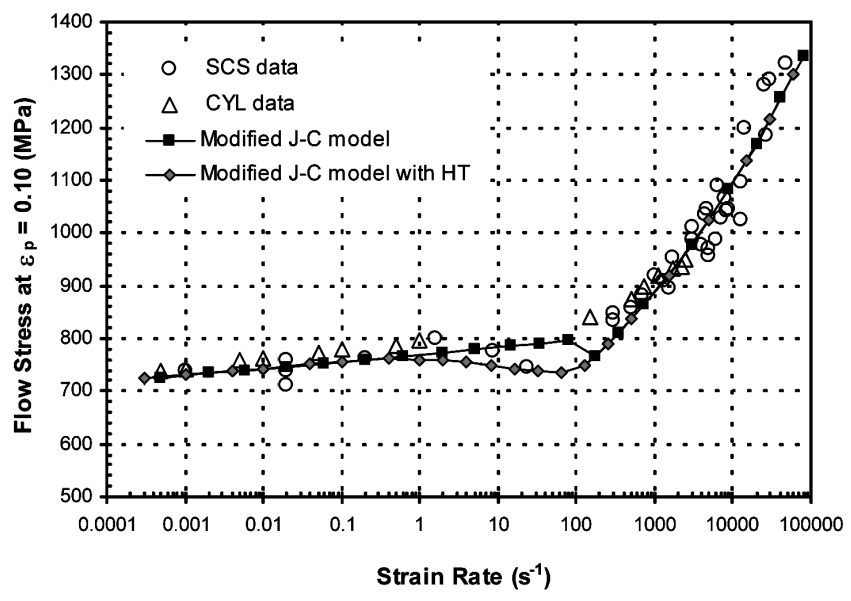

Fig. $11-$ Variation of flow stress at $\varepsilon_{p}=0.10$ as a function of the strain rate. Note that the modified Johnson-Cook model with and without heat transfer (HT) considerations (Appendix B) has the capability to represent experimental data over a very large strain rate range. 
where $S$ is a smooth step function described in Appendix B (Eq. [B5]), $\dot{\varepsilon}_{A}$ is a critical strain rate at which the rate of heat generation is comparable to the rate of heat loss, and $s_{A}$ is a scaling factor that describes the range of transition. Here, $s_{A}$ was taken to be 0.6 in order to set a transition range of three orders of magnitude, as suggested by the experimental data of Dixon and Parry, ${ }^{[24]}$ with $\dot{\varepsilon}_{A}$ being in the center of this transition region. An expression for the approximate estimation of the critical strain rate $\dot{\varepsilon}_{A}$ has been derived by Frost and Ashby ${ }^{[25]}$ based on a conventional compression test arrangement in which a cylindrical specimen of radius $R$ is sandwiched between two parallel steel loading platens. Their (approximate) equation is

$$
\dot{\varepsilon}_{A}=4 n k / R^{2}\left(\frac{\partial \sigma}{\partial T}\right)_{\varepsilon, \dot{\varepsilon}}
$$

where $n$ is the work-hardening exponent ( $n=0.32$ for 1018 steel, Table I), $k$ is the thermal conductivity of the specimen $(k=51.8 \mathrm{~W} / \mathrm{m} \mathrm{K}), \sigma$ is flow stress, $\varepsilon$ is equivalent plastic strain, and $T$ is temperature. Equation [6] yields a value of $\dot{\varepsilon}_{A}=27 \mathrm{~s}^{-1}$ for 1018 steel using $R=2.54 \mathrm{~mm}$, the typical groove width of SCS geometry, and $\partial \sigma / \partial T \approx 0.4$ $\times 10^{6} \mathrm{~N} / \mathrm{m}^{2} \mathrm{~K}$. With these parameters, the variation of $\eta$ in Eq. [5] is shown in Figure 12. Finally, the modified Johnson-Cook model can be coupled with Eqs. [3], [5], and [6] to account for the effect of heat transfer from specimen to the surroundings during the experiment. The predictions of this new form that recognizes the presence of a finite range of transition from isothermal to adiabatic deformation process is also presented in Figure 11 and referred to as the modified Johnson-Cook model with heat transfer (HT) (denoted by solid diamonds). It should be noted that the small dip in the flow stress $v s$ logarithmic strain rate data of Figure 11 between $10^{0}$ and $10^{2} \mathrm{~s}^{-1}$ is also observed in the experimental data reported by Follansbee ${ }^{[28]}$ for various fcc steels and that the heat-transfer correction made to the modified Johnson-Cook model successfully captures this characteristic trend of experimental data in the transient region connecting quasi-static and dynamic deformation regimes.

Another effect related to strain softening is that of the localization of dynamic plastic deformation in the form of shear bands. Such localized bands of deformation have been reported to form in cold-rolled 1018 steel, by Hartley et al. ${ }^{[15]}$ and also by Duffy and Chi. ${ }^{[6]}$ The first article mentioned previously monitored temperature changes and reports a noticeable increase in temperature close to fracture. In the second article, a high-speed camera was used to monitor the local

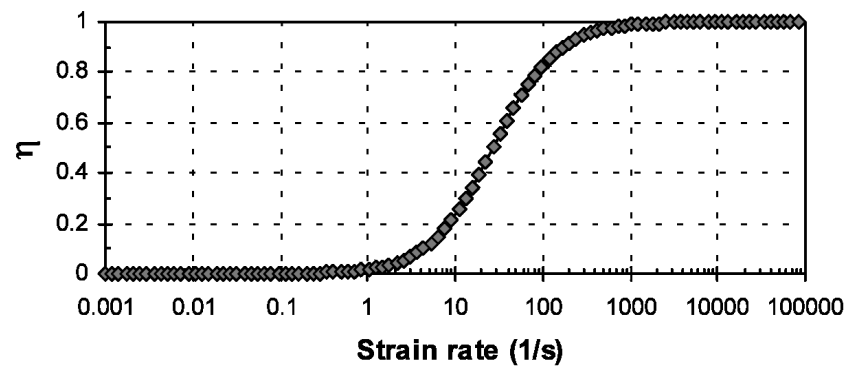

Fig. 12-Variation of $\eta$ as a function of strain rate, which, by definition, is the fraction of heat retained in the deforming body. deformation pattern of a scribed network of lines. The highspeed photographs show the evolution of the shear deformation pattern from a rather homogeneous state at small plastic strains into an increasingly localized shear band toward final fracture. The entire localization process develops while the material exhibits strain softening. Our experiments were not aimed at sorting the relative influence of thermal and shear localization induced softening. However, at this stage, it appears that since localization is reported to develop at the early stages of plastic deformation in this material before the development of significant temperature change, the role of shear localization is probably instrumental in the overall softening process.

In the present work, mechanical as well as microstructural aspects of the shear deformation have been characterized for the SCS geometry. However, at this stage, one cannot draw firm conclusions as to the critical conditions at which the localization starts, in terms of strain or strain rate. On the other hand, this study shows that shear deformation and localization of this material comprises several distinct features. Localization can appear as relatively wide transition bands on both sides of the deformed gage of the SCS specimen (Figure 6). At higher strain rates, these bands can alternatively become much narrower, while the local strain increases markedly (Figure 7). Finally, secondary narrow shear bands can be observed to form in an evenly spaced array across the gage width (Figure 7(c)). It is important to note that all these stages are not observed simultaneously in one specimen, thus suggesting a specific chronology, as follows. First, shear deformation proceeds homogeneously, and the onset of localization corresponds to the formation of the two wide deformation bands at the edges. As deformation progresses, local shear strain increases, thus reducing the width of these bands. Finally, secondary patterns of fine shear bands form across the gage width, shortly before specimen fractures. In this case, the final fracture pattern bears a close relationship with the secondary bands, leading to step formation. It is evident that additional careful microstructural characterization is needed to pinpoint the onset of shear banding and ascertain the proposed shear band evolution. Yet, to the best of our knowledge, there is a scarcity of detailed reports about similar shear band evolution, and it is felt that an important underlying factor must be the hardening of the material and its variations across the gage width, as evidenced by the microhardness measurements reported here.

\section{CONCLUSIONS}

The large-strain constitutive behavior of cold-rolled 1018 steel has been characterized at strain rates ranging from $\dot{\varepsilon}=1 \times 10^{-3}$ to $5 \times 10^{4} \mathrm{~s}^{-1}$, using the SCSs. The following conclusions can be drawn from the present study.

1. The technique based on the SCS yields results that are quite comparable to those obtained using other techniques, such as uniaxial compression or torsion tests.

2. A wide range of strain rates is conveniently explored in a seamless manner using single specimen geometry (SCS) and loading technique (compression). 
3. The SCS technique allows the attainment of higher strain rates when compared with other testing methods, thus bridging the gaps between conventional quasi-static compression and Kolsky (split Hopkinson) pressure bar technique and pressure shear experiments.

4. Contrary to uniaxial testing with cylindrical specimens, the SCS technique has the potential to capture the strain softening behavior of cold-rolled 1018 steel, which is in accord with torsion test observations at similar strain rates.

5. Two distinct regimes of strain rates have been observed for which the material is either almost not affected by strain rate variations, or rather strongly dependent on them. The transition limit is observed to be $\dot{\varepsilon} \approx 10^{2} \mathrm{~s}^{-1}$. The Johnson-Cook model has been modified and its parameters have been determined to represent 1018 steel data over a large range of strain rates covering both regimes.

6. Microstructural observation of the deformation patterns in the sheared gage sections suggests the evolution of the localization process, starting by two wide deformation bands at the edges of gage section that subsequently become narrower while concentrating additional shear strain. Shortly before fracture, a secondary array of equidistant narrow shear bands may form, which dictates the final fracture path.

\section{ACKNOWLEDGMENTS}

This work was supported by the Sandia National Laboratories (Livermore, CA), Dr. D.D. Dawson, Scientific Contact, which is gratefully acknowledged. The authors thank Professor A. Gilat, Ohio State University, for sharing the unpublished torsional data on 1018 steel. One of the authors (GR) acknowledges support from the Office of Naval Research Dr. J. Christodoulou, Scientific Officer) for his work on Dynamic Behavior of Steels, which has led to the development of the experimental techniques used in the present research.

\section{APPENDIX A}

The state of stress within the gage section of SCS geometry under uniaxial compression $(P)$ for the cylindrical sections can be approximated in different ways, which is intimately related to the geometry and in particular to the ratio of width $(w)$ to thickness $(t)$ of the gage section. First, one can assume that the gage section is dominated by the uniaxial state of stress, as shown in Figure A1, i.e., $\sigma_{y}=\sigma_{z}=0$. In this case, the state of stress at a point within the gage section is given as follows:

$$
\begin{aligned}
\sigma_{i j} & =\frac{\sigma_{k k}}{3} \delta_{i j}+S_{i j}=\left(\begin{array}{ccc}
\sigma_{x} & \tau_{x y} & 0 \\
\tau_{x y} & 0 & 0 \\
0 & 0 & 0
\end{array}\right) \text { where } \\
\sigma_{x} & =\frac{P}{D t} \cos ^{2} \alpha \\
\tau_{x y} & =\frac{P}{D t} \cos \alpha \sin \alpha
\end{aligned}
$$

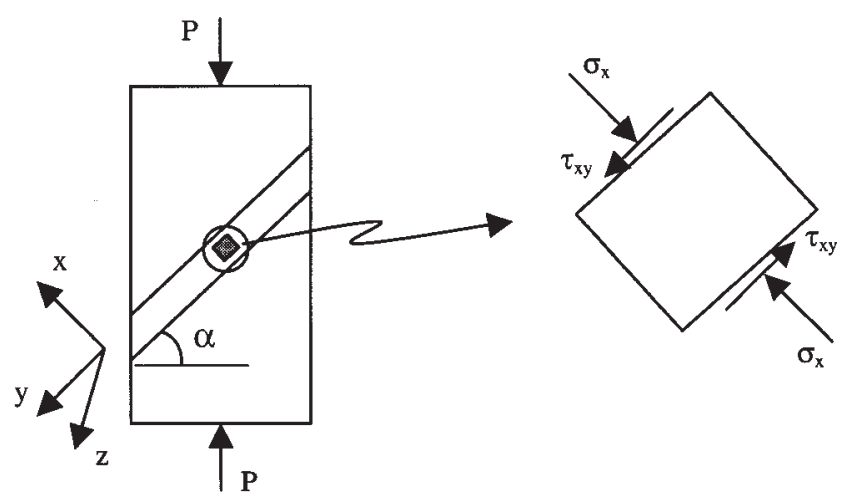

Fig. A1-SCS geometry and the state of stress at a point within the gage section.

and the deviatoric stress tensor

$$
S_{i j}=\frac{P}{D t}\left(\begin{array}{ccc}
\frac{2}{3} \cos ^{2} \alpha & \cos \alpha \sin \alpha & 0 \\
\cos \alpha \sin \alpha & \frac{-\cos ^{2} \alpha}{3} & 0 \\
0 & 0 & \frac{-\cos ^{2} \alpha}{3}
\end{array}\right) \text { [A2] }
$$

The equivalent von Mises stress can be easily found as

$$
\sigma_{e q}=\sqrt{\frac{3}{2} S_{i j} S_{i j}}=\frac{P}{D t} \sqrt{\cos ^{4} \alpha+3 \cos ^{2} \alpha \sin ^{2} \alpha} \text { [A3] }
$$

For $\alpha=45 \mathrm{deg}$, Eq. [A3] becomes

$$
\sigma_{e q}=\sqrt{\frac{3}{2} S_{i j} S_{i j}}=\frac{P}{D t}
$$

The comparison of Eq. [A4] with Eq. [2] suggests that $k_{1}=1$ in Eq. [2].

Second, considering the fact that the gage section is an order of magnitude longer in length with respect to its width and thickness, one can equally well assume the prevalence of plane stress in the gage section, i.e., $\varepsilon_{y}=\sigma_{z}=0$. Thus, the stress tensors in Eq. [A1] become

$$
\sigma_{i j}=\left(\begin{array}{ccc}
\sigma_{x} & \tau_{x y} & 0 \\
\tau_{x y} & \nu \sigma_{x} & 0 \\
0 & 0 & 0
\end{array}\right) \quad \text { and }
$$

$$
S_{i j}=\frac{P}{D t}\left(\begin{array}{ccc}
\frac{2-v}{3} \cos ^{2} \alpha & \cos \alpha \sin \alpha & 0 \\
\cos \alpha \sin \alpha & \frac{2 v-1}{3} \cos ^{2} \alpha & 0 \\
0 & 0 & \frac{-1-v}{3} \cos ^{2} \alpha
\end{array}\right)
$$


Thus, equivalent von Mises stress, in this case, is given by

$$
\begin{aligned}
\sigma_{e q} & =\sqrt{\frac{3}{2} S_{i j} S_{i j}} \\
& =\sqrt{\cos ^{4} \alpha\left(v^{2}-v+1\right)+3 \cos ^{2} \alpha \sin ^{2} \alpha} \frac{P}{D t}
\end{aligned}
$$

which, for $\alpha=45 \mathrm{deg}$ and $v=1 / 2$ (incompressible during plastic deformation), yields

$$
\sigma_{e q}=0.968 \frac{P}{D t}
$$

The comparison of Eq. [A7] with Eq. [2] suggests that $k_{1}=0.968$ in Eq. [2]. It should be noted that the equivalent stresses in uniaxial stress and plane stress conditions differ from each other only by 3 pct.

Finally, one can also argue that the continuity of material at the upper and lower boundaries of the gage section gives rise to a state of uniaxial strain, i.e., $\varepsilon_{y}=\varepsilon_{z}=0$, at least in regions close to gage boundaries. For this case, the stress tensors are given as follows:

$$
\sigma_{i j}=\left(\begin{array}{ccc}
\sigma_{x} & \tau_{x y} & 0 \\
\tau_{x y} & \frac{v}{1-v} \sigma_{x} & 0 \\
0 & 0 & \frac{v}{1-v} \sigma_{x}
\end{array}\right) \text { and }
$$

$S_{i j}=\frac{P}{D t}\left(\begin{array}{ccc}\frac{2(1-2 v)}{3(1-v)} \cos ^{2} \alpha & \cos \alpha \sin \alpha & 0 \\ \cos \alpha \sin \alpha & \frac{2 v-1}{3(1-v)} \cos ^{2} \alpha & 0 \\ 0 & 0 & \frac{2 v-1}{3(1-v)} \cos ^{2} \alpha\end{array}\right)$

and the equivalent von Mises stress becomes

$$
\begin{aligned}
\sigma_{e q} & =\sqrt{\frac{3}{2} S_{i j} S_{i j}} \\
& =\sqrt{\frac{(1-2 v)^{2}}{(1-v)^{2}} \cos ^{4} \alpha+3 \cos ^{2} \alpha \sin ^{2} \alpha} \frac{P}{D t}
\end{aligned}
$$

which, for $\alpha=45 \mathrm{deg}$ and $v=1 / 2$, yields

$$
\sigma_{e q}=0.866 \frac{P}{D t}
$$

The comparison of Eq. [A10] with Eq. [2] suggests that $k_{1}$ $=0.866$ in Eq. [2]. It should be noted that the stress state in this last case (Eq. [A8]) reduces to a state of pure shear superimposed by a hydrostatic stress component for $\nu=1 / 2$, as in the pressure-shear experiments of Clifton and Klopp. ${ }^{[3]}$

\section{APPENDIX B}

The Johnson-Cook constitutive equation ${ }^{[18]}$ has the following form with three distinct terms that define, respectively, the strain hardening, strain rate dependence, and temperature dependence of the flow stress,

$$
\begin{gathered}
\sigma=\left(\sigma_{0}+B \varepsilon^{n}\right)\left(1+C \ln \frac{\dot{\varepsilon}}{\dot{\varepsilon}_{0}}\right)\left(1-T_{*}{ }^{p}\right), \\
\text { where } T_{*}=\frac{T-T_{r}}{T_{m}-T_{r}}
\end{gathered}
$$

where $\sigma_{0}$ is the yield stress of material at a reference temperature of $T_{r}$ and strain rate of $\dot{\varepsilon}_{0} ; B, n, C$, and $p$ are the model parameters that should be determined by experiments; and $T_{m}$ is the absolute melting temperature of the material under consideration. The Johnson-Cook equation is a phenomenological constitutive model that is commonly used in numerical codes to predict the large-strain, high-strain-rate, and high-temperature flow stress of materials (e.g., Meyers $\left.{ }^{[1]}\right)$. However, with this form, the model is limited to a specific region in which the flow stress is a linear function of the logarithm of strain rate and, therefore, should be modified to capture the nonlinear material response over a large strain rate interval such as shown in Figure B1. Figure B1 is a generalized schematic representation of material behavior where there are two distinct regions with different strain rate dependence of the flow stress, which is similar to the behavior observed for 1018 steel, as shown in Figure 4.

By following the general approach commonly used in the investigation of strain-rate sensitivity of materials (e.g., Tong et al., ${ }^{[26]}$ Estrin et al. ${ }^{[27]}$ ), we will describe the rate dependence of flow stress by power-law relations as in the following:

$\sigma=\sigma_{\mathrm{o}}\left(\frac{\dot{\varepsilon}}{\dot{\varepsilon}_{01}}\right)^{n_{1}}$ in region 1 , and $\sigma=\sigma_{\mathrm{o}}\left(\frac{\dot{\varepsilon}}{\dot{\varepsilon}_{02}}\right)^{n_{2}}$ in region 2

where $n_{i}$ is the rate sensitivity of the flow stress $\sigma$, and $\dot{\varepsilon}_{0 i}$ is the reference strain rate $(i=1,2)$. Here, we will describe $\sigma$ in Eq. [B2] as the yield stress of material. On the other hand, at zero plastic strain and reference temperature, the Johnson-Cook model gives the yield stress as

$$
\sigma=\sigma_{0}\left(1+C \ln \frac{\dot{\varepsilon}}{\dot{\varepsilon}_{0}}\right)
$$

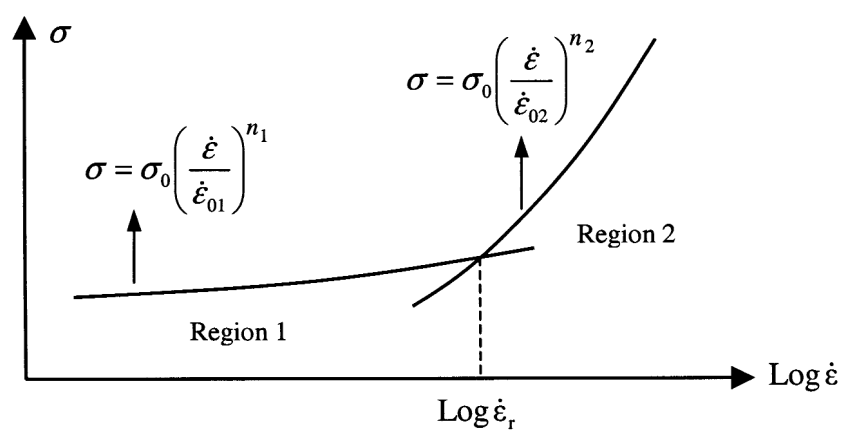

Fig. B1-Schematics of the typical strain rate dependence of flow stress. 
Differentiating Eqs. [B2] and [B3] with respect to $\partial \ln \dot{\varepsilon}$ and equating them yields

$$
C_{i}=n_{i}\left(\frac{\dot{\varepsilon}}{\dot{\varepsilon}_{0 i}}\right)^{n_{i}}
$$

Naturally, the prediction of the generalized response in Figure B1 requires the information about two distinct rate-dependent strain rate hardening factors $\left(C_{1}, C_{2}\right)$ and transition strain rate $\left(\dot{\varepsilon}_{t}\right)$ to be implemented into the model. In order to make this implementation in a smooth manner, a continuous function is proposed, as follows:

$$
S\left(\dot{\varepsilon}, s_{1}, s_{2}, r\right)=s_{1}+\frac{s_{2}-s_{1}}{2}\left[1+\tanh \left(s \ln \frac{\dot{\varepsilon}}{r}\right)\right]
$$

This function gives $s_{1}$ when $\dot{\varepsilon}<r$ and gives $s_{2}$ when $r<\dot{\varepsilon}$, with a smooth and continuous variation around the transition value $r$. The transition interval can be kept wide or very short depending on the proper choice of a scaling factor given by $s$, for which a value of $s=4 / \ln (1+0.01 \delta)$ provides $99.9 \mathrm{pct}$ of the transition from one state to another to occur within $\pm \delta$ pct vicinity of the transition value $r$. In this study, the value of $s=400$ is adopted for $\delta=1$. Thus, the Johnson-Cook equation in Eq. [B1] can be modified by re-defining the model parameter $C$ as a continuous function of strain rate:

$$
C=S\left(\dot{\varepsilon}, C_{1}, C_{2}, \dot{\varepsilon}_{t}\right)
$$

Furthermore, the consideration of continuity in the yield stress of material at the end and the beginning of the sequential regions in Figure B1 provides one with the complementary relation for reference strain rates in each particular region, i.e., $\dot{\varepsilon}_{01}$ and $\dot{\varepsilon}_{02}$. In this case, if $\sigma_{0}$ in Eq. [B1] is the yield stress of material at a reference strain rate of $\dot{\varepsilon}_{01}$ for the first region, the reference strain rate for the second region is given by

$$
\dot{\varepsilon}_{02}=\left(\dot{\varepsilon}_{01}\right)^{n_{1} / n_{2}}\left(\dot{\varepsilon}_{t}\right)^{\left(n_{2}-n_{1}\right) / n_{2}}
$$

Thus, the Johnson-Cook model has two interdependent reference strain rates for the generalized situation and this information is provided to the model by re-defining the reference strain rate in Eq. [B1] as a function of strain rate; i.e.,

$$
\dot{\varepsilon}_{0}=S\left(\dot{\varepsilon}, \dot{\varepsilon}_{01}, \dot{\varepsilon}_{02}, \dot{\varepsilon}_{t}\right)
$$

With these modifications, the Johnson-Cook model can be used to predict material response over a wide range of strain rates where multiple regions with different strain rate hardening trends exist. It should be noted that each new region requires two extra parameters, which basically define the strain rate hardening and the transition strain rate. Thus, the number of parameters that should be determined by experiments in the modified Johnson-Cook model increases from 5 to 7 , which are $\sigma_{0}, B, n, n_{1}, n_{2}, \dot{\varepsilon}_{t}$, and $p$.

\section{REFERENCES}

1. M.A. Meyers: Dynamic Behavior of Materials, John Wiley \& Sons, New York, NY, 1994.

2. H. Kolsky: P. Phys. Soc. London, 1949, vol. 62-B, pp. 676-700.

3. R.J. Clifton and R.W. Klopp: Metals Handbook, 9th ed., ASM INTERNATIONAL, Metals Park, OH, 1986, vol. 8, p. 230.

4. J. Duffy, J.D. Campbell, and R.H. Hawley: J. Appl. Mech, 1971, vol. 38, pp. 83-91.

5. A. Marchand and J. Duffy: J. Mech. Phys. Solids, 1988, vol. 36(3), pp. $251-83$

6. J. Duffy and Y.C. Chi: Mater. Sci. Eng. A-Struct., 1992, vol. 157(2), pp. $195-210$

7. A. Gilat and X.R. Wu: Int. J. Plasticity, 1997, vols. 6-7, pp. 611-32.

8. A. Gilat and C.S. Cheng: Int. J. Plasticity, 2002, vols. 5-6, pp. 787-99.

9. M.A. Meyers, L.W. Meyer, K.S. Vecchio, and U. Andrade: J. Phys. IV, 1991, vol. 1 (C3), pp. 11-17.

10. D. Rittel, S. Lee, and G. Ravichandran: Exp. Mech., 2002, vol. 42(1), pp. 58-64.

11. D. Rittel, G. Ravichandran, and S. Lee: Mech. Mater., 2002, vol. 34(10), pp. $627-42$.

12. G.I. Taylor and H. Quinney: P. R. Soc. London, 1934, vol. A143, pp. 307-26.

13. Y. Bai and B. Dodd: Adiabatic Shear Localization: Occurrence, Theories, and Applications, Pergamon Press, Oxford, UK, 1992.

14. L.S. Costin and J. Duffy: J. Eng. Mater.-Trans. ASME, 1979, vol. 101(3), pp. 258-64.

15. K.A. Hartley, J. Duffy, and R.H. Hawley: J. Mech. Phys. Solids, 1987, vol. 35(3), pp. 283-301.

16. R. Kapoor and S. Nemat-Nasser: Mech. Mater., 1998 vol. 27, pp. 1-12.

17. C.S. Cheng: Ph.D. Thesis, The Ohio State University, Columbus, $\mathrm{OH}$, 1999.

18. G.R. Johnson and W.H. Cook: Proc. 7th Int. Symp. on Ballistics, The Hague, The Netherlands, 1983, pp. 541-47.

19. G.T. Gray III: ASM Handbook, ASM INTERNATIONAL, Metals Park, OH, 2000, vol. 8, pp. 462-76.

20. Y.B. Xu, Z.G. Wang, X.L. Huang, D. Xing, and Y.L. Bai: Mater. Sci. Eng., 1989, vol. A114, pp. 81-87.

21. J.D. Campbell and W.G. Ferguson: Phil. Mag., 1970, vol. 21, pp. 63-75.

22. J.J. Mason, A.J. Rosakis, and G. Ravichandran: Mech. Mater., 1994, vol. 17, pp. 135-45.

23. D. Rittel: Mech. Mater., 1999, vol. 31(2), pp. 131-39.

24. P.R. Dixon and D.J. Parry: J. Phys. IV, 1991, vol. 1(C3), pp. 85-92.

25. H.J. Frost and M.F. Ashby: Deformation-Mechanism Maps, Pergamon Press, Oxford, United Kingdom, 1982, pp. 120-23.

26. W. Tong, R.J. Clifton, and S. Huang: J. Mech. Phys. Solids, 1992, vol. 40(6), pp. 1251-94.

27. Y. Estrin, A. Molinari, and S. Mercier: J. Eng. Mater. and Tech.-Trans. ASME, 1997, vol. 119(4), pp. 322-31.

28. P.S. Follansbee: in Metallurgical Applications of Shock-Wave and HighStrain-Rate Phenomena, M.L. Murr, K.P. Staudhammer, and M.A. Meyers, eds., Marcel Dekker Inc., New York, NY, 1986. pp. 451-79. 